\title{
Postnatal women's experiences of management of depressive symptoms: a qualitative study
}

\author{
Pauline Slade, C Jane Morrell, Anna Rigby, Karen Ricci, \\ Janet Spittlehouse and Traolach S Brugha
}

\begin{abstract}
Background

Postnatal depression is a public health problem requiring intervention. To provide effective care, information is needed on the experiences of those with high levels of depressive symptoms who are offered and accept, or decline, psychological intervention postnatally.
\end{abstract}

Aim

To provide the first integrated in-depth exploration of postnatal women's experiences of the identification and management of symptoms of depression and the offer and acceptance of postnatal care by health visitors taking part in the PoNDER trial.

\section{Setting}

General practice: primary care within the former Trent regional health authority, England.

\section{Method}

Thirty women with 6-week Edinburgh Postnatal Depression Scale (EPDS) scores $\geq 18$ and probable depression completed semi-structured interviews. All women had taken part in the Post-Natal Depression Economic Evaluation and Randomised controlled (PoNDER) trial where intervention group health visitors received training in identification of depressive symptoms and provided psychologically informed sessions based on cognitive-behavioural therapy or person-centred counselling principles.

\section{Results}

When accepted, psychological sessions were experienced as positive, effective, and 'ideal care'. Women approved of using the EPDS but did not understand the health visitor's role in supporting women. Seeking help and accepting sessions depended on women's perspectives of their health visitor as an individual.

\section{Conclusion}

Women's experience of their health visitors providing psychological sessions to help with postnatal depressive symptoms is highly positive. Women will better accept support from health visitors if they recognise their role in postnatal depression and find them easy to relate to on personal matters. There is a case for specific enhancement of interpersonal skills in health visiting, or alternatively offering a choice of health visitors to women.

\section{Keywords}

intervention; postnatal depression; primary health care; psychological.

\section{INTRODUCTION}

Postnatal depression is a public health issue affecting $10-13 \%$ of women, ${ }^{1}$ and influencing the woman herself, her partner, ${ }^{2}$ her relationship with her infant, ${ }^{3}$ and consequent child development. ${ }^{4}$ Support for women is provided mainly within primary care. Health visitors have been encouraged to see identification of postnatal depression and subsequent provision of support as a part of their role. ${ }^{5}$ The most commonly used method for identifying symptoms is the Edinburgh Postnatal Depression Scale (EPDS), ${ }^{6}$ alongside a health visitor's clinical judgement.

Postnatal women with depressive symptoms are reluctant to seek help, partly because of the perceived stigma and wishing to be seen as 'good mothers'. ' Issues of acceptability of the EPDS, ${ }^{8-11}$ and barriers to

P Slade, MSc, PhD, professor of clinical psychology and consultant clinical psychologist, Clinical Psychology Unit, Department of Psychology, University of Sheffield, Sheffield. CJ Morrell, MPhil, PhD, HV, RGN, associate professor in health research, School of Nursing, Midwifery and Physiotherapy, Faculty of Medicine and Health Sciences, University of Nottingham, Nottingham. A Rigby, MSc, research psychologist, Fieldhead Hospital, CarePathways and Packages Project, Wakefield. J Spittlehouse, BSc, research psychologist, University of Otago, Christchurch, CHALICE Project, New Zealand. TS Brugha, MD (NUI), FRCPsych, professor of psychiatry; K Ricci, $M A$, research psychologist, University of Leicester, Clinical Division of Psychiatry, Leicester General Hospital, Leicester.

Address for correspondence

Professor Pauline Slade, Clinical Psychology Unit, Department of Psychology University of Sheffield, Sheffield, S102TN.E-mail: p.slade@sheffield.ac.uk

Submitted: 22 April 2010; Editor's response: 1 June 2010; final acceptance: 16 June 2010.

(C)British Journal of General Practice

This is the full-length article (published online 1 November 2010) of an abridged version published in print. Cite this article as: BrJ Gen Pract 2010; DOI: 10.3399/bjgp10X532611. 
disclosure, ${ }^{7}$ help-seeking, ${ }^{12}$ and referral ${ }^{13}$ have been explored, but little is known about the perspectives of women with depression regarding postnatal interventions when offered.

Cross-cultural studies of postnatal depression have established postnatal women's wishes for opportunities to address mood disturbances through talking therapies rather than medication. ${ }^{14}$ For women with low levels of depressive symptoms, factors that may make 'listening visits' a positive experience are: a belief in the medical model for postnatal depression, a good relationship with the health visitor, and clear and flexible choices and arrangements. ${ }^{15}$

\section{Rationale for the study}

While information is available about women's experiences of postnatal depression, reasons why postnatal women find help-seeking difficult, and professionals' perspectives on the provision of diagnosis of postnatal depression,,$^{16}$ little is known about the experiences of women with high levels of depressive symptoms of being offered and accepting or declining psychological support. Better understanding of these processes, from women's own perspectives, would enhance effective service provision. This study provided a unique opportunity to explore the views of women with high levels of depressive symptoms.

\section{Summary of the PoNDER trial}

Internationally, the Post-Natal Depression Economic Evaluation and Randomised controlled (PoNDER) trial is the largest postnatal depression intervention study, with 4084 consenting women participating..$^{17}$ It used a pragmatic randomised cluster design and demonstrated the clinical ${ }^{17}$ and cost-effectiveness ${ }^{18}$ of additional training for health visitors. Women were recruited to the trial by their health visitor if they were registered with participating (and therefore randomised) GP practices. As this was a pragmatic trial, it aimed to be as inclusive as possible. The only criteria were that women were required to be aged 18 years or over, and to have no severe or enduring mental health problems. Health visitors were asked to post such women a research information leaflet and a consent form at 36 weeks' pregnancy.

Health visitors in the intervention group completed a day of training in the identification of depression using the EPDS and clinical assessment, and then 5 days of training delivered over 4 weeks focusing on the development of cognitive-behavioural therapy or person-centred intervention skills. This was followed by four 3-hour reflective practice sessions at weeks 6 , 10,14 , and 18, and access to regular supervision sessions. A common element of the training, preceding specific approach-based skills, involved developing

\section{How this fits in}

Providing intervention for women with postnatal depression is important for public health. Little is known about what influences women who are depressed postnatally to accept help if it is offered. This study suggests that health visitors' personal skills in forming relationships and women's understanding of the health visitors' role with mothers and not just babies may be key factors.

empathic relationships. Two experienced psychotherapist practitioner trainers delivered the training, which was carried out according to a manual, ${ }^{19}$ and was agreed by a training reference group, comprising experienced academically-based psychotherapy and counselling trainers. In addition, health visitors were asked to audio record their intervention sessions (with the women's consent), so that session treatment fidelity could be checked. Good adherence levels were achieved, although the number of tapes was limited. Further details of the training are available elsewhere. ${ }^{18}$

The training helped to develop health visitors' skills in developing empathic relationships, identify postnatal depressive symptoms systematically, and deliver psychologically informed sessions.

Identification of women with postnatal depression included clinical assessment by the health visitor and the use of the EPDS. Sessions with health visitors involved psychologically informed interventions using cognitive-behavioural therapy techniques or personcentred counselling principles, and women could be offered up to eight 1-hour sessions. The control group received health visitor usual care. Women in the intervention group with a 6 -week EPDS score $\geq 12$ had a reduction in depressive symptoms as measured by the EPDS at both 6 and 12 months postnatally when compared to the control. At 6 months, $33.9 \%$ of women scored above 12 on the EPDS in the intervention group compared to $45.6 \%$ in the control group. This difference of $11.7 \%$ was statistically significant $(P=0.036){ }^{18}$ This improvement occurred despite $40 \%$ of women declining the sessions offered to them. Also, the intervention group, across the full spectrum of 6-week EPDS scores, showed more positive outcomes than the control group.

This qualitative study of women's experiences helped to provide some explanation for the quantitative trial findings. To understand the experiences of those women with significant depressive symptoms, the sample comprised all intervention and control women with an EPDS score $\geq 18$, indicating a high probability of clinical depression.

This paper provides the first integrated in-depth exploration of depressed women's experiences of identification of depressive symptoms postnatally and the provision of postnatal care. 


\begin{tabular}{|c|c|c|c|c|}
\hline & PCA & $\mathrm{CBA}$ & CG & Total \\
\hline$n$ & 11 & 10 & 9 & 30 \\
\hline \multicolumn{5}{|l|}{ Age, years } \\
\hline $18-25$ & 2 & 2 & 3 & 7 \\
\hline $26-35$ & 6 & 5 & 4 & 15 \\
\hline $36-45$ & 3 & 3 & $0^{\mathrm{a}}$ & 6 \\
\hline Single parent, $n$ & 1 & 1 & 1 & 3 \\
\hline Partnered, $n$ & 6 & 3 & 1 & 10 \\
\hline First child, $n$ & 7 & 8 & 6 & 21 \\
\hline GP prescribed antidepressant, $n$ & 6 & 5 & 7 & 18 \\
\hline
\end{tabular}

${ }^{a}$ Missing $\mathrm{n}=2$. $P C A=$ person-centred approach. $C B A=$ cognitive-behavioural approach. $C G=$ control group. questions, while allowing further, richer detail in the form of additional themes or subthemes to emerge from the data. It contrasts with purely exploratory methods which would not have been appropriate here, as there were some specific questions to address.

Templates are made up of codes that are hierarchically organised; the highest-level codes are the broad themes, while lower-level codes are more narrowly focused aspects of the broader theme. The original prespecified template focused on the main research questions: women's experiences relating to identification of symptoms, information about postnatal depression from the health visitor, women's experience of the EPDS, and women's route to support together with their experiences of support, including what would have constituted their ideal support.

Three interviewers developed the codes together, modified them after successive readings of the transcripts, and then produced a final structured representation of themes, and the relationships between them, which adequately reflected the whole dataset. Checks for contradictory information were incorporated. The evidence for the final themes and subthemes was independently verified by a researcher experienced in qualitative methodology, with assistance from another researcher.

\section{RESULTS}

The final template of themes is shown in Box 1. A theme that detailed women's experiences of postnatal depression confirms findings ${ }^{21}$ around actual signs of emotional distress, losing identity as separate from the baby, pressures of motherhood and breastfeeding, and are therefore not reported in detail here.

\section{Theme 1: seeking help (Box 2)}

Help-seeking styles. Several processes appeared to influence help-seeking behaviour. For some women this involved recognising their difficulties themselves and acting on this. Others sought help indirectly, typically via presenting a concern about the infant or their children's illnesses, as a 'way in' to seeking help for themselves. Most frequently, however, women responded to (strong) advice from others, family or friends, that they needed help.

Table 2. Intervention group $(n=21)$ by CBA or PCA and support received.

PCA, $n=11$

CBA, $n=10$ Total

\begin{tabular}{llll}
\hline Offered sessions & 5 patients (4 antidepressants) & 5 patients (4 antidepressants) & $10(48 \%)$ \\
\hline Offered and declined sessions & 1 patients (0 antidepressants) & 2 patients (1 antidepressants) & $3(14 \%)$ \\
\hline Not offered sessions & 5 patients (3 antidepressants) & 3 patients (0 antidepressants) & $8(38 \%)$ \\
\hline
\end{tabular}

$P C A=$ person-centred approach. $C B A=$ cognitive-behavioural approach 
Barriers to accessing help. Women found the helpseeking process difficult, especially 'admitting' to needing help and the implication of failure. Women wanted to present a 'coping' image. They feared appearing unable to look after their infant and what others might think or do. Worryingly, they were afraid that their infant might be taken away from them. There was perceived extra pressure from other mothers appearing to 'do it right'.

The perception of the woman's health visitor could be a significant barrier. Critically for some women, their perception of their health visitor as someone they could not relate to emotionally or who was not well known to them led them to decline the health visitor's offer of sessions. The importance of women's perceptions of health visitors' role also emerges in relation to themes 2 and 3 .

\section{Theme 2: roles and relationships (Box 3)}

Completing the EPDS. The interviewers asked the women about use of the EPDS 'questionnaire'. The

\section{Box 1. Final template of themes and subthemes.}

\section{Theme 1: seeking help}

1.1 Help-seeking styles

1.1.1 Self

1.1.2 Through consulting on other issues

1.1.3 Others taking action

1.2 Barriers to help

1.2.1 Presenting a coping image

1.2.2 Perception of their health visitor in relation to seeking help

\section{Theme 2: roles and relationships}

2.1 Completing the Edinburgh Postnatal Depression Scale

2.2 Information about postnatal depression

2.3 Relationship with the health visitor

2.4 Perceptions of the health visitor's role

\section{Theme 3: experiences of intervention or support}

3.1 Cognitive-behavioural approach

3.2 Person-centred approach

3.3 Treatment as usual: the control group

3.4 Ideal support

\section{Box 2. Theme 1: seeking help.}

\subsection{Help-seeking styles}

1.1.1 Self

'... Driving on the M25 and I've actually had the thought as regards "Wouldn't it be easier if I just drove off?", and at that point I thought "No, something's not quite right here, don't be an idiot ...".' (control, ID 21)

1.1.2 Through consulting on other issues

'... so I called him [GP] out ... I called him for [my infant] but I did want to talk to him at the same time ... but I used the excuse of him coming to see [my infant].' (CBA - NOS, ID 22)

\subsubsection{Others taking action}

'It was sort of my partner saying to me "Right if you don't go l'm basically making you an appointment, you are going, don't sweep it under the carpet, you know, you can't just keep feeling like this".' (CBA - AS, ID 12)

\subsection{Barriers to help}

1.2.1 Presenting a coping image

'I didn't want anyone's help to be honest after I had [my previous child]. I was so frightened that people would think I couldn't cope and take her off me.' (PCA - AS, ID 20)

'I remember thinking "I don't want her to think I'm not coping", which is stupid really because I wasn't ... Especially ... you're stood in the middle of the surgery, with like six mums around you with six newborns and you just think, well I'm hardly gonna turn round and go, you know, "I'm really not f***ing coping, help".' (CBA - AS, ID 17)

1.2.2 Perception of their health visitor in relation to seeking help

'... So I think she wasn't as person-centred and she didn't really have the people skills to manage, you know, she could have, sort of offered advice and support in a much more supportive way instead of "Well you haven't done this, you haven't done that", and her tone was all wrong as well.' (PCA - DS, ID 11)

'I did ask for support but I didn't really get any. And the health visitor's response ... "Well you seem like you're doing alright", which kind of closes it off doesn't it then?' (control, ID 7)

'I didn't feel like talking to her. I didn't really know her that well so ...' (CBA - DS, ID 4) 


\section{Box 3. Theme 2: roles and relationships.}

2.1 Completing the Edinburgh Postnatal Depression Scale

'... at least when [the health visitor] came with the Edinburgh scale, at least somebody knows that at that particular moment you feel dreadful.' (PCA - NOS, ID 8)

2.2 Information about postnatal depression

'She [health visitor] did tell me exactly what it was all about [postnatal depression].' (control, ID 24)

'I didn't really know much about it to be honest ... nothing from a ... professional point of view.' (PCA - AS, ID 3)

2.3 Relationship with the health visitor

'Very good, absolutely like a really good honest relationship and I feel very like I trust her [health visitor] and that you know that I can confide in her and talk to her really about everything that's sort of like going on since l've had the baby ...' (CBA - AS, ID 10)

'So she [the health visitor] was like supportive and kept in contact quite a lot, ringing me to see if I was ok and if I needed to talk, she was there sort of thing.'

This woman felt continuity was important:

'I always ask to speak to [health visitor] because I know her and it's easier really if you know somebody.' (PCA - NOS, ID 30)

'... I don't feel I had any support either as a new mum or emotionally or whatever else I don't think there was a relationship to the point where both [my partner] and I would dread her coming out to weigh him ... and as I say at the point where we were told ... that we'd had a new health visitor, thank god for that, hooray.' (control, ID 21)

2.4 Perceptions of the health visitor's role

'I thought it was just snooping ... that's what I thought health visiting was. It's trying to see if you're doing anything wrong with your children for social services. I didn't realise that it's ... not about that, it's about keeping families together, not tearing them apart ...' (PCA - AS, ID 20)

'Oh I think before the visits you don't really know them ... if your baby's alright and you're alright ... you only see them at assessments and things. I now know in the future if I've got any concerns, you know, I could just pick up the phone ... yeah more confident towards them.' (CBA - AS, ID 12)

'I don't really know what their job is ... Nobody gave me like the parameters of this role of the health visitor and so, I think if that happened then you'd ... know what the function was ... and sort of be able to use them better.' (PCA - DS, ID 11)

:... At first I was using them [the health visitors] as I thought they were meant to be used, which was asking questions all the time and checking things out with them to make sure I was right, you know basic people to talk to as some kind of support. To at least make me feel as though I was doing things right, and if I wasn't how to change it and do it slightly different. But then I soon realised that they weren't giving me any help whatsoever ...'

She went on to describe health vistors as being:

'... more like your Nanna telling you what to do.' (PCA - DS, ID 28)

This woman was very positive about the support from her GP.

findings reflected the variability of health visitors' administrative styles, with some women being unaware a questionnaire was being used. Only one woman was entirely negative about the use of the EPDS, and most welcomed the opportunity to talk about their feelings. Furthermore, the interviewees also appreciated the use of the EPDS because it shifted the focus onto themselves rather than concentrating on the infant.

Information about postnatal depression. There were variations in how well informed women were about postnatal depression, ranging from having extensive information and good understanding, to having no information at all, or confusion with the 'baby blues'.
Relationship with the health visitor. The quality of a woman's relationship with her health visitor, and the health visitor's interpersonal skills appeared crucial in determining the quality of experiences of postnatal care. Women who accepted psychologically informed sessions tended to be extremely positive about their relationship with their health visitor. Women in the control group who were well supported were also positive about their relationship with their health visitor. Negative comments about relationships with the health visitor occurred in intervention group women who had either not been offered or declined sessions, or control group women who perceived themselves as receiving little or no support. 
Perceptions of the health visitor's role. The final subtheme related to women's perceptions of the role of the health visitor. Women spoke about the health visitor's role in relation to the infant, and typically did not understand that part of the health visitor role included providing emotional care for the mother. For some women, this understanding changed after they had accepted sessions.

\section{Box 4. Theme 3: experiences of intervention or support.}

3.1 Cognitive-behavioural approach

'But it was just talking about the relationship with my boyfriend and how I felt about baby and just talking through my week. What had helped, what hadn't helped. What I could try and do to make myself feel better. Just looking on positives rather than negatives ... Find the thought patterns that led down the wrong way.' (CBA - AS, ID 17)

'We've analysed all the reasons why l've been down and depressed, how to sort of, challenge negative thoughts...'

The woman describes how the health visitor helped her:

'... The worst time ... I couldn't think about anything logically, whereas she sort of like focused me ...'

It was also important for her that:

'I don't feel like I'm being judged at all.' (CBA - AS, ID 10)

One woman not offered sessions said

'... I needed your help then not now.'

She also went on to say:

'You know, you need the contact, somebody to talk to face to face, this is me, this is what's happening to me now ...' (CBA - NOS, ID 18)

3.2 Person-centred approach

'Yeah ... she did help me through; she made me understand why I was feeling like that ... I don't think I would be feeling like I am now if [the health visitor] didn't have come ... because I have mainly talked I think it's helped me in the fact that I can understand what I'm feeling rather than, er getting suggestions from other people ... It's helped me work out what I should do to make myself feel better.' (PCA - AS, ID 29) 'I owe so much to her [the health visitor] and it's really brought out of me to be open and everything.' (PCA - AS, ID 31)

'... [The health visitor] explained about listening visits ... but I actually struggled to talk cos I'd rather be challenged. l'd rather be asked questions ... so I did explain to [the health visitor] after the seventh [visit] that I'm struggling to talk so it's not benefiting me ... even though [the health visitor] were ever so good.' (PCA AS, ID 26)

3.3 Treatment as usual: the control group

'She is very supportive and really it's good having someone that you can talk to about how you really feel.' She went on to comment that:

'She is not critical in any way.'

'She always make another appointment with me so I always knew that I was going to be seeing her again fairly soon.' (control, ID 14)

'... And we just carried on talking and I were getting better as weeks went on.' (control, ID 19)

'They're always having to rush off.'

She had told the health visitor:

'... How I were feeling and things like that, and I told her what had happened in t'past and erm, she said a few things and that were about it and I haven't heard nowt from her since.' (control, ID 9)

'I didn't really feel like the health visitor was very sympathetic really ... and she didn't really pick up on indicators I was trying to give her. "I'm not actually" and that's even quite difficult to admit to yourself isn't it, that you're not coping with your baby?' (control, ID 7)

3.4 Ideal support

'... I just hope from this trial that health visitors will be able to visit as they did me and more if needed, cos I do think it's important ...' (PCA - AS, ID 3)

'She was there from the first time she came round ... I can't say I would have changed it.' (PCA - AS, ID 31) 'It would have been better for me if someone had said "I think we need to talk about this, do you want to speak to somebody?" or maybe some counselling ... or maybe to get it off my chest with [the health visitor], she's pushed for time a lot, we do questionnaires and that but obviously didn't have the time for me to sit there and say oh this has happened and that has happened.' (CBA - NOS, ID 1)

'HVs who aren't like your Nanna, they like have counselling training and ... they're able to listen to you ... All you want is someone to actually listen to what you're saying, even if it's complete crap and it's all coming out wrong. You just want someone to say "It's alright, sit down and I'll listen to what you've got to say". That would do you the world of good and I think it would actually stop people from developing worse symptoms because people just won't talk about it.' (PCA - NOS, ID 28) 


\section{Theme 3: experiences of intervention or support (Box 4)}

Women who received any psychologically informed sessions reported strongly positive experiences, placing particular emphasis on the importance of having the opportunity for one-to-one discussions with the health visitor. The sessions were not only 'acceptable' to women, but also very successful. Subthemes related to specific experiences of a cognitive-behavioural approach to intervention, person-centred approach to intervention, usual care, and ideas relating to ideal care.

Cognitive-behavioural and person-centred approaches to intervention. The cognitive-behavioural approach group who received sessions commented on the specific components of the approach; in particular, the value of identifying what made them feel better and worse, and the role of thinking patterns, as well as positive comments on the effect of the sessions. For the person-centred approach intervention group, three of the five women made a comment on the nature of the approach and on the benefits of the sessions. Only one woman found this approach difficult, perhaps indicating the need for tailoring therapeutic approaches to the needs of the individual.

Intervention group women who were not offered the health visitor sessions had more varied experiences, and, perhaps unsurprisingly, were dissatisfied with the amount of input. Overall they were positive about the support they did receive, which tended to be more practical in nature and focused on the infant. One woman not offered sessions had a more negative experience, as she reported saying to her health visitor that she had really needed her help but it was not forthcoming. Where women declined help they felt they did not really know the health visitor or that she was not the sort of person they could talk with.

Treatment as usual: the control group. Control group women experienced usual but very varied health visitor practice. Those women who had experienced support from the health visitor (for example, home visits or encouragement by the health visitor to phone if needed) reported the most positive experiences. Several control group women who reported their health visitor was aware that they were having difficulties received little or no support at all. One woman who unusually saw two health visitors felt the first had dismissed her feelings and put them down to: 'being a new mum', while she had a more positive experience with the second health visitor. This highlighted the variability in the service of usual care, even when there was awareness of distress. This contrasts with the more positive comments from the intervention group women who received the sessions.
Ideal support. Almost all women receiving the health visitor sessions viewed this support as ideal and would not have changed this in any way. One woman in the intervention group wanted 'proper counselling' as she felt it had all been too informal. Those women not in receipt of health visitor sessions thought some form of counselling, an opportunity to talk, and someone understanding should be offered to women in their situation.

\section{DISCUSSION}

\section{Summary of main findings}

The findings unique to this study were that the health visitor sessions, when accepted, were highly valued, and seen as beneficial and as 'ideal support'. Women's ideals of support invariably involved the availability of someone understanding and sympathetic with whom to talk, however limited this availability might be. In addition, this study indicates that reasons for women declining health visitor sessions were often that the women had ongoing dissatisfaction with their relationship with the health visitor.

This is the first study to report that symptom identification through using the EPDS helped women to view the health visitor as 'there for them', as well as the infant, as depressed women were often unaware of the health visitor's role in supporting mothers.

Being open about mental health problems postnatally was difficult for women who were depressed, confirming previous work. ${ }^{12}$ This difficulty was linked to feelings of shame, and fears of being seen 'not to cope'. The women also indicated that they had received variable amounts of information about postnatal depression, also confirming previous work. ${ }^{12}$

The overarching theme in these interviews concerned women's perceptions of the personal qualities of the healthcare professional, which was likely to be linked to the health visitor's training in developing skills in establishing supportive relationships. These perceptions appeared to determine women's willingness to be open about sensitive issues, and influenced whether health visitors' offers of psychologically informed sessions were accepted.

The role of communication skills in general practice in influencing the effectiveness of systematic interventions for depression in primary care has been explored previously. ${ }^{22}$ The important contextual difference for postnatal women is that the experience of postnatal depression is associated with shame and fear of 'not coping' as a mother. This may relate to the fact that having had a baby is generally regarded as a positive event, so women are expected to respond positively. It is particularly important that all those providing health care for women who have postnatal depression, adopt an accepting, non-judgemental approach. 


\section{Strengths and limitations of the study}

There was a high participation rate, with 30/39 (77\%) eligible women agreeing to participate in the study. The findings for women with higher levels of depressive symptoms (who had not undergone formal diagnosis of depression) may not extrapolate to women with lower levels of symptoms. The methodology could have been potentially strengthened by using participant validation.

\section{Comparisons with existing literature}

This study confirms the findings of earlier work on barriers to help-seeking and fears of being seen as not coping, ${ }^{12}$ and on the acceptability of the EPDS in women with high levels of symptoms, supporting a recent systematic review. ${ }^{11}$

\section{Implications for future research and clinical practice}

Postnatal women will not only accept psychologically informed sessions to help with depression, but also value them and see them as beneficial and ideal when provided by a health visitor skilled in interpersonal relationships. Women's help-seeking and acceptance of support may be facilitated by special training for health visitors in relationship skills. Consistent information is needed about postnatal depression and about the health visitor's role in providing support for mothers and not only care for the infant. While in the PoNDER trial the quality of engagement was measured by women and health visitors to compare the two approaches within the intervention group, ${ }^{18}$ there was no pre-post comparison of interpersonal skills, nor was there any equivalent measurement in the control group. Future research needs to evaluate whether training such as that provided in the PoNDER trial specifically improves engagement skills, and whether this is a key element in facilitating helpseeking behaviour, and, consequently, improvements for women with postnatal depression.

\section{Funding body}

The trial was commissioned, funded, and sponsored by the NHS R\&D Health Technology Assessment Programme, 99/33/51.

\section{Ethics committee}

Ethical approval was provided by Trent MREC on 29 January 2003: reference number 02/4/108.

\section{Competing interests}

The authors have stated that there are none.

\section{Discuss this article}

Contribute and read comments about this article on the Discussion Forum: http://www.rcgp.org.uk/bjgp-discuss

\section{REFERENCES}

1. Gaynes BN, Gavin N, Meltzer-Brody S, et al. Perinatal depression: prevalence, screening accuracy, and screening outcomes. Evidence Report/Technol Assess No. 119. Chapel Hill, NC: University of North Carolina Evidence-based Center, 2005.

2. Ballard CG, Davies R, Cullen PC, et al. Prevalence of postnatal psychiatric morbidity on mothers and fathers. Br J Psychiatry 1994; 164(6): 782-788.

3. Martins C, Gaffan EA. Effects of early maternal depression on pattern of infant mother attachment: a meta-analytic investigation. J Child Psychol Psychiatry 2000; 41(6): 737-746.

4. Murray L, Cooper PJ. The role of infant and maternal factors in postpartum depression, mother-infant interactions, and infant outcomes. In: Murray L, Cooper P (eds). Postpartum depression and child development. London: The Guilford Press, 1997; 111-135.

5. Department of Health. Into the mainstream, implementation plan: mainstreaming gender and women's mental health. London: Department of Health, 2003.

6. Cox JL, Holden JM, Sagovsky R. Detection of postnatal depression. Development of the 10-item Edinburgh Postnatal Depression Scale. Br J Psychiatry 1987; 150: 782-786.

7. Chew-Graham CA, Sharp D, Chamberlain E, et al. Disclosure of symptoms of postnatal depression, the perspectives of heath professionals and women: a qualitative study. BMC Family Practice 2009; 10: 7.

8. Gemmill AW, Leigh B, Ericksen J, Milgrom J. A survey of the clinical acceptability of screening for postnatal depression in depressed and non-depressed women. BMC Public Health 2006; 6: 211.

9. Poole P, Mason L, Osborn T. Women's views of being screened for postnatal depression. Community Pract 2006; 79(11): 363-367.

10. Shakespeare J, Blake F, Garcia J. A qualitative study of the acceptability of routine screening of postnatal women using the Edinburgh Postnatal Depression Scale. Br J Gen Pract 2003; 53(493): 614-619.

11. Hewitt CE, Gilbody SM, Brealey S, et al. Methods to identify postnatal depression in primary care: an integrated evidence synthesis and value of information analysis. Health Technol Assess 2009; 13(36): 1-145, 147-230.

12. Dennis C L, Chung-Lee L. Postpartum depression help seeking barriers and maternal treatment preferences: a qualitative systematic review. Birth 2006; 33(4): 323-331.

13. Sword W, Busser D, Gannaan R, et al. Women's care seeking experiences after referral for postpartum depression. Qual Health Res 2008; 18(9): 1162-1173.

14. Oates MR, Cox JL, Neema S, et al. Postnatal depression across countries and cultures: a qualitative study. Br J Psychiatry 2004; 46: s10-s16.

15. Shakespeare J Blake F, Garcia J. How do women with postnatal depression experience listening visits in primary care? A qualitative interview study. J Reprod Infant Psychol 2006; 24(2): 149-162.

16. Chew-Graham C, Chamberlain E, Turner K, et al. GPs' and health visitors' views on the diagnosis and management of postnatal depression. Br J Gen Pract 2008; 58(548): 169-176.

17. Morrell CJ, Slade P, Warner R, et al. Clinical effectiveness of health visitor training in psychologically informed interventions for depression in postnatal women: pragmatic cluster randomised trial in primary care. BMJ 2009; 338: a3045.

18. Morrell CJ, Warner R, Slade P, et al. Psychological interventions fo postnatal depression: cluster randomised trial and economic evaluation. The PoNDER trial. Health Technol Assess 2009; 13(30): iii-iv, xi-xiii, 1-153.

19. Morrell CJ, Ricketts T, Tudor K, et al. Training health visitors in cognitive behavioural and person-centred approaches for depression in postnatal women as part of a cluster randomised trial and economic evaluation in primary care: the PoNDER trial. Prim Health Care Res Dev 2010: doi:10.1017/S1463423610000344

20. King, N. Template analysis. In: Symon G, Cassell C (eds). Qualitative methods and analysis in organizational research. London: Sage, 1998.

21. Beck CT. Postpartum depression: a metasynthesis. Qual Health Res 2002; 12(4): 453-472.

22. Van Os TWDP, van den Brink RHS, Tiemens BG, et al. Communicative skills of general practitioners augment the effectiveness of guideline-based depression treatment. J Affect Disord 2005; 84(1): 43-54 


\section{Appendix 1. Interview schedule for the semi structured interview.}

\section{Introductions}

- Purpose: to add qualitative information to the data source.

- Assurances of confidentiality, except in cases of potential harm.

- Importance of taping/consent form for data analysis.

\section{6-8 week history}

- Some women suffer from postnatal depression at the 6-8 week postnatal period. How did you feel at this time?

- Did you think you were depressed? Anything else?

- Did anyone tell you that you had postnatal depression?

- Were you breastfeeding at the time?

- Were you prescribed antidepressants?

- Who suggested you take them?

- Did you obtain them from the chemist/take them/for how many weeks?

- How helpful do you feel they were?

\section{Health visitor}

- What did the health visitor say to you about postnatal depression?

- If she thought you were depressed, what led her to believe you were depressed?

- Did your health visitor offer you one-to-one visits? Did you accept the offered visits?

- If you did not accept the offer of these visits, please can you explain why?

- About how many one-to-one visits did you have from the health visitor?

- How useful did you find the visits?

For cognitive-behavioural approach ask about the homework/person-centred approach 'listening' role of the health visitor

- Good points/bad points?

- What do you think about the health visitor's support overall?

- How helpful was the health visitor?

- How do you feel now?

- What was your previous relationship with the health visitor compared with what it's like now?

\section{Other support}

- Did you receive support from others at this time? Was it formal/informal? If formal, who suggested it?

- What kind of support did you get from your GP? Did you have a diagnosis of postnatal depression?

\section{Future}

- What sort of support would you like to see in the future for women with postnatal depression? 